\title{
Visual Function for Driving in Diabetic Macular Edema and Retinal Vein Occlusion Post-Stabilization with Anti-Vascular Endothelial Growth Factor
}

\author{
Donald R Nixon ${ }^{1-4}$ \\ Nicholas Flinn' \\ 'Trimed Eye Centre, Barrie, Ontario, \\ L4M 4S5, Canada; ${ }^{2}$ Department of \\ Surgery, Royal Victoria Regional Health \\ Centre, Barrie, Ontario, Canada; \\ ${ }^{3}$ Department of Surgery, Soldiers \\ Memorial Hospital, Orillia, Ontario, \\ Canada; ${ }^{4}$ Department of Surgery, \\ Northern Ontario School of Medicine \\ NOSM, Sudbury, Ontario, Canada
}

Purpose: What is the level of visual function in patients with diabetic macular edema (DME) and retinal vein occlusion (RVO) post-stabilization with anti-vascular endothelial growth factor?

Patients and Methods: This observational non-controlled single center study evaluated visual function in two patient populations with macular edema 25 with diabetic macular edema and 25 with retinal vein occlusion treated following standard protocol of anti-VEGF therapy post- stabilization.

Results: A total of 68 eyes from 50 patients were analyzed including 18 bilateral and 7 unilateral diabetic macular edema, 14 patients with central and 11 with branch retinal vein occlusion. The mean age was $69 \pm 11$ years and $64 \%$ were male. In the RVO group: LogMAR BCVA was $0.12 \pm 0.13$ compared to the unaffected eye $0.04 \pm 0.05(\mathrm{P}=<0.01)$, contrast sensitivity in the treated eye was $1.69 \pm 0.21 \log$ units compared to $1.84 \pm 0.15 \log$ units in the unaffected eye $(\mathrm{p}=<0.01)$, the ganglion cell volume was $0.88 \pm 0.15 \mathrm{~mm}^{3}$ in the treated eye compared to $1.04 \pm 0.1 \mathrm{~mm}^{3}$ in the unaffected eye $(\mathrm{P}=<0.01)$. In the diabetic macular edema group: LogMAR BCVA was $0.17 \pm 0.13$, contrast sensitivity in the treated eye was $1.16 \pm 0.21$ $\log$ units compared to the normal population $1.92 \pm 0.8 \log$ units $(\mathrm{p}=<0.01)$, the ganglion cell volume was $0.94 \pm 0.14 \mathrm{~mm}^{3}$ in the treated eye compared to $1.03 \pm 0.12 \mathrm{~mm}^{3}$ in the normal population $(\mathrm{P}=<0.001)$. In both groups a majority of treated eyes retained visual acuity $\geq+0.4$ LogMAR (diabetic macular edema 95\%, RVO 96\%) however contrast sensitivity was more than two standard deviations below the normal population mean in a majority of treated eyes in both groups (diabetic macular edema 88\% RVO 64\%).

Conclusion: Impairment in contrast sensitivity in both groups could impact activities of daily living including driving and should prompt questions about how we advise patients regarding their level of function and the potential limitations/restrictions that should be placed on such activities.

Keywords: contrast sensitivity, residual deficit, functional impairment

\section{Introduction}

Visual function assesses the overall interaction with the external environment through the visual system. Testing for this includes visual acuity (VA), contrast sensitivity (CS), visual fields and colour vision. Although there is overlap between each ability, they are unique and can be affected separately in retinal diseases. ${ }^{1-3}$
Correspondence: Donald R Nixon

Trimed Eye Centre Barrie Ontario, 190

Cundles Road East Suite 100c, Barrie,

Ontario, L4M 4S5, Canada

$\mathrm{Tel}+$ I 7057373737

Email Trimedeyedoc@gmail.com 
Two specific abilities, visual acuity and contrast sensitivity, have been well recognized to be affected in diabetic macular edema (DME) and retinal vein occlusion (RVO) and can be tested in several different ways. ${ }^{4} \mathrm{VA}$ is tested at $100 \%$ contrast and the optotype reduces in size to determine what arc angle the patient can resolve. With contrast sensitivity testing, both the level of contrast and optotype size can change depending on the testing modality used. The extent to which VA and contrast sensitivity can be affected, as well as the level of recovery with therapy, depends on the duration and severity of the disease. ${ }^{5}$ Visual acuity has been well documented to improve with anti-VEGF therapy in both $\mathrm{RVO}^{6}$ and $\mathrm{DME}{ }^{7}$ but fewer studies have included contrast sensitivity as an outcome parameter. ${ }^{8}$ Contrast sensitivity is important for a number of functions including mobility, reading speed, quality of life, and driving. Symptoms of reduced contrast sensitivity are most prevalent in conditions of low light, ${ }^{9}$ fog, or reflective glare. ${ }^{10} \mathrm{~A}$ decrease in contrast sensitivity can lead to a loss of spatial awareness, mobility, and can increase the risk of injuries including motor vehicle accidents. This condition is not a direct indication of poor or weakening VA. It is possible to have normal VA while also experiencing reduced contrast sensitivity; therefore testing photopic VA alone is not a good predictor of low contrast function and performance. ${ }^{10}$

In some countries efforts are made to vary testing parameters for a driving licence such as background lighting and contrast by assessing visual function outdoors with a poor performance resulting in a failure of the certification exam. Unfortunately although this is a more realistic test environment there is no agreed to standards for contrast sensitivity thresholds and level of luminance so few countries include testing in different levels of luminance or environments. ${ }^{9}$ Multiple testing technologies are available to evaluate contrast sensitivity with factors such as cost, testing time, and patient's abilities considered in the technology chosen. A component of this study is to use and compare two commercially available testing methods, the Pelli-Robson and CamBlobs. The Pelli-Robson requires literacy skills and compared to CamBlobs, requires more technician time, longer duration, and is more costly. The letter sequences of the Pelli-Robson chart are organized into groups of three (triplets) with two triplets per line. Within each triplet, all letters have the same contrast. The contrast decreases from one triplet to the next. The CamBlobs, now known as the "SpotChecks"TM", is an inexpensive alternative that can be administered as a self-test and requires no literacy skills, providing rapid results. The test, made up of 4 columns of 25 rectangles, requires the subject to mark the location of a $9 \mathrm{~mm}$ circular grey disk in each rectangle progressing down the page. Each test is a single use test where patients test themselves by marking an " $\mathrm{x}$ " on each contrast target they see. Testing is intuitive; individuals can complete the test within 2-3 minutes with rapid results as scoring is easy and quick. The test sheet itself then becomes the record for the patient file.

Concerns about visual performance is relevant today as diabetes in the adult population has increased almost fourfold from 1980 to 2014 due to population growth, increased prevalence, and aging in the population. DME is the most common cause of decreased VA with up to $10 \%$ of diabetic patients affected. ${ }^{10,14}$ RVO is second only to diabetic retinopathy as a cause of visual loss due to retinal vascular disease. There are two forms; branch retinal vein occlusion BRVO and central retinal vein occlusion CRVO which can be further subdivided to ischemic and nonischemic with macular edema (ME) being the main cause of the deterioration of visual acuity. ${ }^{11,13} \mathrm{~A}$ significant percentage of both of these populations drive, and the impact of impairment in CS could be associated increased driver related accidents. ${ }^{9,21,22,26}$

This study was designed to focus on outcome levels of VA and CS in the two groups, DME and RVO poststabilization of $\mathrm{ME}$, as well as compare and contrast these results and their possible impact on future performance. In addition, high-resolution spectral domain OCT testing was used at the time of resolution of the retinal edema in both groups, to generate a quantitative assessment of the ganglion cell layer (GCL) and ganglion cell volume (GCV). This was done to try to get a better understanding of the lasting effects of these inner retinal vascular diseases and its relationship to visual function. ${ }^{12}$

\section{Patients and Methods}

This institutional review board (IRB)-Approved (IRB Services, Aurora, ON, Canada) (CR00153811) prospective, observational, non-controlled, single center study was conducted at Tri Med Laser Eye Center, Barrie Ontario between March and June of 2018. A total of 25 patients with diabetic macular edema, and 25 patients with either CRVO or BRVO, were enrolled if they met the inclusion and exclusion criteria listed in Table 1. The study was conducted in accordance with the principles of the Declaration of Helsinki and in compliance with Good 
Table I Inclusion and Exclusion Criteria

\begin{tabular}{|l|l|}
\hline Inclusion Criteria & Exclusion Criteria \\
\hline Informed consent & Uncontrolled IOP $(>25 \mathrm{mmHg})$ \\
\hline$\geq 18$ years of age & Prior retinal scaring, cataracts, or vitreous hemorrhage \\
\hline Ability to complete study & Intravitreal steroid treatment within prior 6 months \\
\hline Diagnosis of center involving DME or RVO & MI, TIA, or CVA within prior 90 days \\
\hline Currently treated with Aflibercept or Ranibizumab & Diagnosis of Glaucoma \\
\hline "Dry" SD-OCT (macula thickness less than $315 \mu \mathrm{m}$ and reestablishment of foveal pit) & \\
\hline Baseline fluorescein angiography & \\
\hline VA $>6 / 30$ & \\
\hline
\end{tabular}

Clinical Practice and applicable regulatory requirements. Written informed consent was obtained from all participants before study enrollment.

The primary endpoints were the VA, CS, and ganglion cell volume of the treated eyes as compared to either the normal database for contrast sensitivity ${ }^{17,19}$ and $\mathrm{GCV}^{13}$ for DME eyes or, for subjects in the RVO arm of the trial, the unaffected eye and the normative database were used as the controls. Additionally, the correlation in log contrast scores obtained for both treated and untreated eyes using Pelli-Robson contrast sensitivity scoring and Camblobs contrast sensitivity were examined to establish if a correlation exists in the log contrast scores obtained using these testing methods.

Each of the patient populations that satisfied the criteria for intervention for $\mathrm{DME}^{7}$ and $\mathrm{RVO}^{6}$ received a loading dose $^{6,7}$ with either ranibizumab or aflibercept and were followed with treatment for at least 6 months. Patients in each group were considered stable for enrollment if they had at least two consecutive 8 weeks intervals of stable VA and less than a $5 \%$ change in central subfield thickness (CST) as measured by spectral domain OCT. Subjects were enrolled only after resolution of macular edema was confirmed with spectral domain OCT, specifically a macular thickness $<315 \mu \mathrm{m}$, corresponding to normal value +2 SDs: $277+(2 \times 19) \mu \mathrm{m}$ and the reestablishment of the foveal pit were used as markers for the resolution of fluid. $^{3}$

Eligible subjects were tested monocularly for contrast sensitivity using the Pelli-Robson chart and the CamBolbs contrast sensitivity testing system, as well as high-resolution OCT and visual acuity. The testing with Pelli-Robson was completed at $1 \mathrm{~m}$ using the "per letter" method of scoring. ${ }^{14,15}$ The CamBlobs was self-administered, taken in good lighting, at a comfortable reading distance.

Monocular BCVA was recorded using the Snellen chart and converted to the logarithm of minimum angle of resolution (logMAR) equivalents for analysis.

OCT measures, including CRT and GCV, were measured using SD-OCT (Heidelberg Spectralis, Heidelberg Engineering In, Vista, CA USA) and compared to a normative database for this system. ${ }^{16}$

\section{Statistical Analysis}

Statistical analysis was completed following the completion of the subjects using $\mathrm{R}$ version 3.4.0. Visual function statistical analysis included mean LogMAR BCVA and mean contrast sensitivity comparing study population to either unaffected eye or normal population. ${ }^{17}$ Anatomical statistical analysis included mean ganglion cell layer (GCL) volume comparing study population to either unaffected eye or normal population. If not otherwise stated, all values are presented as mean \pm SD. Comparison of population means was completed using Welch's unequal variances $t$-tests. A P-value $<0.05$ was considered statically significant. The normal data for CamBlobs contrast sensitivity was used for the study. ${ }^{16,17}$

Pearson correlation of log contrast scores obtained with Pelli-Robson and Camblobs tests were calculated for all subject eyes, including both treated and untreated.

\section{Results}

\section{Characteristics of Study Population}

A total of 68 eyes from 50 patients were included in the analysis; this included 14 CRVO, 11 BRVO and 18 bilateral and 7 unilateral DME patients. In the DME group 
three eyes had focal laser but none within the previous year. In the RVO group the untreated eye had a complete oculo-visual assessment and was determined to be normal. The mean age of the patients in the DME group was $69 \pm 11$ years and $76 \%$ were male, in the RVO group mean age was $66 \pm 11$ years and $52 \%$ were male. All patients completed the required testing and no adverse events were reported during their participation in the study.

\section{Measures of Visual Function and Anatomic Outcomes CamBlobs vs Pelli Robson}

Monocular testing of all subject eyes for CamBlobs and Pelli Robson contrast sensitivity were moderately correlated with consistently higher values observed for Camblobs testing $\mathrm{r}(98)=0.65, \mathrm{p}<0.05$.

\section{RVO}

The OCT measured GCL volume of treated eye RVO patients was $0.88 \pm 0.15 \mathrm{~mm}^{3}$ while the unaffected eye was $1.04 \pm 0.1 \mathrm{~mm}^{3}$. The difference in mean GCL volume between the unaffected and treated eye in this study was $0.16 \mathrm{~mm}^{3}(\mathrm{P}=<0.01$, Figure 1).

The LogMAR BCVA in the treated eye was $0.12 \pm 0.13$ while in the unaffected eye it was $0.04 \pm 0.05$. The difference between the treated and unaffected eyes was 0.08 $(\mathrm{P}=<0.01$, Figure 2).

Camblobs contrast sensitivity in the treated eye was 1.69 $\pm 0.21 \log$ units compared to $1.84 \pm 0.15 \log$ units in the unaffected eye, a difference of $0.15 \log$ units $(\mathrm{P}=<0.01$, Figure 3)

Pelli-Robson contrast sensitivity in the treated eye was $1.52 \pm 0.17 \log$ units compared to $1.64 \pm 0.12 \log$ units in the

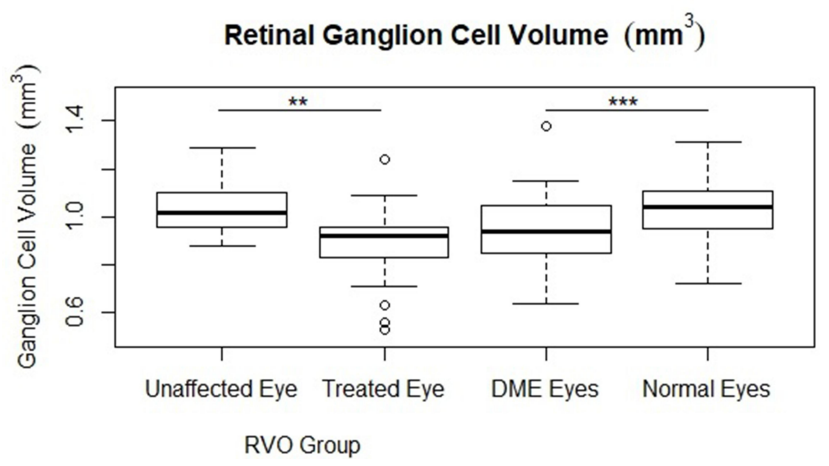

Figure I OCT measured retinal ganglion cell volume $\left(\mathrm{mm}^{3}\right)$ in all groups, black bar represents median value, open circles are outliers, statistical significance is noted above relevant groups $(* * p<0.01$, *** $p<0.001)$.

Abbreviations: RVO, retinal vein occlusion; DME, diabetic macular edema.

\section{RVO LogMAR BCVA}

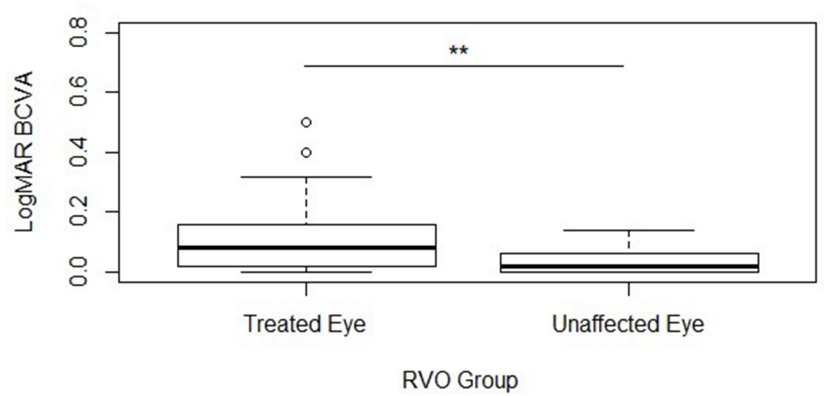

Figure 2 LogMAR BCVA in RVO (retinal vein occlusion) patient's treated and unaffected eye, black bar is the median value, open circles are outliers, statistical significance is noted above relevant groups $(* * p<0.01)$.

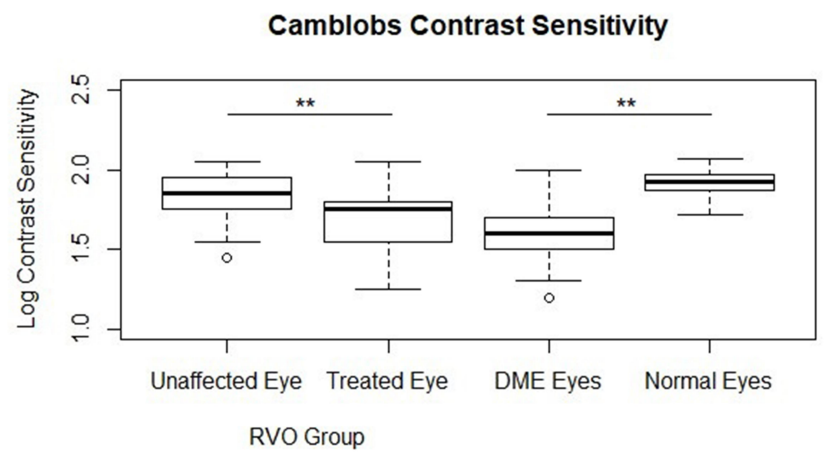

Figure 3 Camblobs contrast sensitivity in all groups, black bar is the median value, open circles are outliers, statistical significance is noted above relevant groups $(* * p<0.01)$.

Abbreviations: RVO, retinal vein occlusion; DME, diabetic macular edema.

unaffected eye, a difference of $0.12 \log$ units $(\mathrm{P}=<0.01$, Figure 4).

There was a weak relationship between Log contrast sensitivity and GCL volume in the treated and unaffected eyes $\left(\mathrm{R}^{2}=0.19, \mathrm{P}=<0.001\right)$.

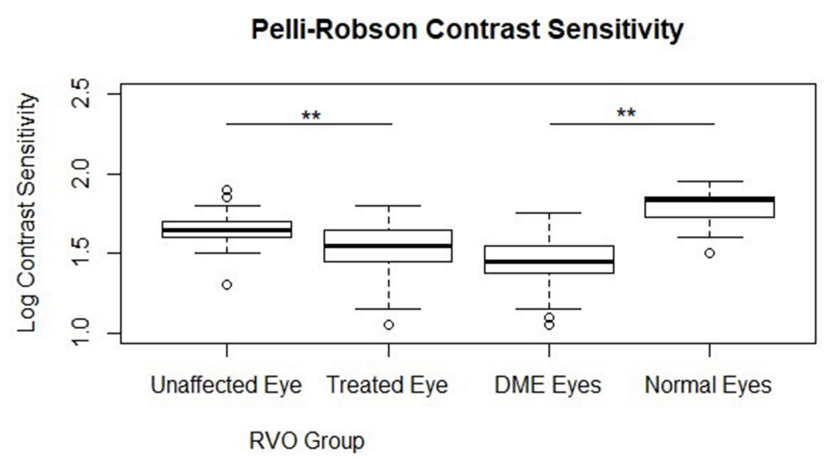

Figure 4 Pelli-Robson contrast sensitivity in all groups, black bar is the median value, open circles are outliers, statistical significance is noted above relevant groups $(* * \mathrm{p}<0.01)$.

Abbreviations: RVO, retinal vein occlusion; DME, diabetic macular edema. 


\section{Diabetic Macular Edema}

OCT measured GCL volume in diabetic macular edema subjects was $0.94 \pm 0.14 \mathrm{~mm}^{3}$ compared to $1.03 \pm 0.12 \mathrm{~mm} 3$ in the normal population data, a difference of $0.09 \mathrm{~mm} 3$ $(\mathrm{P}=<0.001$, Figure 1).

Camblobs contrast sensitivity in diabetic macular edema eyes was $1.58 \pm 0.16 \log$ units compared to 1.92 $\pm 0.8 \mathrm{log}$ units in the normal population, a difference of $0.34 \log$ units $(\mathrm{P}=<0.01$, Figure 3$)$.

Pelli-Robson contrast sensitivity in diabetic macular edema eyes was $1.46 \pm 0.15 \log$ units compared to 1.79 $\pm 0.10 \log$ units in the normal population, a difference of $0.33 \log$ units $(\mathrm{P}=<0.01$, Figure 4$)$.

LogMAR BCVA in diabetic eyes undergoing treatment was $0.17 \pm 0.13$.

There was a weak relationship between Log contrast sensitivity and GCL volume in the diabetic macular edema eyes $\left(\mathrm{R}^{2}=0.24, \mathrm{P}=<0.01\right)$.

\section{Discussion}

Studies to date have shown impressive treatment results with the advent of anti-VEGF treatment for DME and RVO with a reduction in macular edema and improvement in VA. ${ }^{6,7}$ The majority of patients in these groups can continue to be active, and if individuals had stopped during the acute phase, most can re-establish high acuity tasks such as driving based on our present VA testing thresholds.

In both groups, DME and RVO, the final corrected VA in the affected eye was LogMAR 0.4 or better in a majority of subjects ( $95 \%$ of DME and $96 \%$ of RVO patients). The threshold of 0.4 or better was chosen to comply with the vision standards for driving in Canada. ${ }^{18}$ However, in the same national guidelines, the recommendation for contrast sensitivity testing and its incorporation as a supplement to visual acuity assessments has not been to date followed in any province. ${ }^{18}$ When comparing within the same group there was a significant percentage that had contrast sensitivity loss that was greater than two standard deviations (SD) below the mean (64\% RVO and $88 \%$ diabetic macular edema) using the comparative normative database for DME and the unaffected eye in the RVO population. The monocular testing of contrast sensitivity used both Pelli-Robson and CamBlobs, with the log contrast scores found to be comparable. ${ }^{21}$ The Camblobs test is less expensive and patient-guided, reducing staff time in comparison to the long established Pelli-Robson test. The results of this study suggest that the CamBlobs
(SpotChecks ${ }^{\mathrm{TM}}$ ) provides similar results with a testing method that can be easily implemented in regular clinical practice.

The difference in residual deficit in contrast sensitivity between the DME and RVO groups may be explained by the studies showing patients with diabetic macular edema, diabetic retinopathy without edema, and persons with diabetes without manifest retinopathy, suffer from decreased contrast sensitivity before changes in VA occur as measured on traditional tests. ${ }^{1-3}$ Inner retinal vascular diseases such as diabetic retinopathy and RVO are known to measurably reduce the inner retinal cell layers which may lead to the reduction in contrast sensitivity observed. However, these diseases differ in that with diabetic retinopathy the cause and sequence of damage are multifactorial including metabolic, inflammatory, and vascular, as well as being chronic and progressive in nature. ${ }^{5}$ In contrast, RVO can be considered an acute insult with the degree and duration of the vascular occlusion influencing the overall outcomes. There does appear to be a relationship between a reduction in retinal edema and fluid leakage, as measured by ocular coherence tomography (OCT) with an improvement in VA; however, the amount of improvement and the final outcome has limited predictability. Researchers in ophthalmology focusing on glaucoma are also investigating reduced contrast sensitivity as part of visual functional loss in those with glaucoma. Current studies in glaucoma associated the linkage of loss of contrast sensitivity to loss of ganglion cell layer thickness in the macula; ${ }^{19}$ a physiological parallel can be drawn to diabetic macular edema and RVO patients as they too lose ganglion cells throughout the course of the disease. ${ }^{20}$ It is unclear at this time whether the patients with macular edema that lose contrast sensitivity will regain any of what they have lost once they stabilize with current treatment recommendations on intravitreal anti-VEGF treatment. If contrast sensitivity can be regained following treatment, there are no recommendations available as to when to initiate anti-VEGF treatment if the goal is to preserve or regain contrast sensitivity function. If ganglion cell loss is responsible for the decrease in contrast sensitivity then it is likely that the amount of loss may act as a predictor of how much improvement may occur.

In this study, there was a trend to a reduced volume in the ganglion cell layer volume (GCLV) within the ETDRS template that correlated with the reduction in the contrast sensitivity. A similar relationship did not exist with CRT 
and contrast sensitivity or total retinal volume within the ETDRS testing area as noted in other studies. ${ }^{16}$

In the RVO group there was no difference in GCLV between the normative group and the unaffected eye. There was a statistically significant reduction in the GCLV after stability in the treated eye against the unaffected eye and the normative data. In a similar comparison, there was a statistically significant reduction in GCLV and contrast sensitivity in the treated and stable population of RVO compared to the normative data. In both groups, there was a positive relationship to reduction in contrast sensitivity and GCLV, but results were mixed (strong P-value but weak $\mathrm{r}^{2}$ ). The weak correlation between GCLV reduction with decreased contrast sensitivity in both the DME and RVO groups may be because the reduction in $\mathrm{GC}$ is focal rather than diffuse, so looking at variability in discrete volume thickness or focal testing may be associated with more sensitive identification of ganglion cell loss than total ETDRS template volume. ${ }^{5}$

The major concern generated from this study is the measurable reduction in contrast sensitivity identified in both groups. Historically, it was problematic how to interpret these results; however, now consensus supports that a significant loss in contrast sensitivity does represent a meaningful change in overall visual function. ${ }^{21}$ Using data from recent studies we can conclude that in the diabetic macular edema group in this study there was an average reduction of 0.34 contrast sensitivity Log units, which is equivalent to 17 ETDRS letters or more than 3 lines of acuity. ${ }^{22}$ Although a lower average amount of contrast sensitivity reduction was seen in the RVO arm, there was a 0.15 contrast sensitivity Log unit reduction which is equivalent to 7.5 ETDRS letters or 1.5 lines of acuity. This can have an impact on task performance and quality of life. $^{23,24}$

There are many studies that confirm the independence of changes in VA and contrast sensitivity ${ }^{1,2}$ in retinal diseases and glaucoma. Evidence that VA and contrast sensitivity are affected differently in different medical retinal diseases during anti- VEGF therapy ${ }^{25}$ indicates the need to assess both to fully understand a patient's functional level as it relates to activities of daily living such as driving at the time of stability of retinal thickness. As clinicians, the findings of this study should prompt questions about how we advise patients regarding their level of function and the potential limitations and restrictions they should place on their own activities. We found that while patients had improvements in their VA from their first office visit, they still suffered lasting impairments in contrast sensitivity which can have major consequences in common activities like driving at night or in poor weather. Recent research has tried to quantify the real-world risks of impaired contrast sensitivity and visual function, as it specifically relates to driving, which may guide professionals when advising patients. ${ }^{11}$ However, without wide scale testing and specific enforceable restrictions in place, we must still rely on patients exercising their best judgment when undertaking continued or resumption of driving. Questions arise as to what shared risk do physicians have in caring for these patients that still decide to drive?

There are many questions that remain, such as if ganglion cell loss is responsible for the decrease in contrast sensitivity then will the amount of GC loss act as a predictor for the amount of improvement that may occur following treatment? Will earlier intervention, in cases of diabetic retinopathy and diabetic macular edema delay, reduce, or prevent loss of GCL and therefore preserve contrast sensitivity? What modality would be best to apply contrast sensitivity testing in a non-academic setting to evaluate loss? How far are we away from setting contrast sensitivity levels for licensed activities? Is there a staged approach to developing contrast sensitivity thresholds and their implementation? Ultimately, could a license with limitations be the solution to concerns in relation to contrast sensitivity and road safety, with research and health promotion toward prevention techniques and restoration of contrast sensitivity vision being the primary goal?

\section{Conclusion}

Gathering more information about contrast vision is hindered by the fact that most practitioners do not measure contrast vision because it does not provide actionable information for clinical decisions. This, however, may be a vicious circle; if it is not measured, its clinical significance cannot be assessed. In this study we found that the Camblobs was an easy test to administer, patient friendly, with the results comparable to the Pelli-Robson. Several studies have suggested that low contrast function losses may be a more sensitive detector of early disease than high contrast letter chart losses. ${ }^{11}$ The results of this study are limited by the small sample size retrospective nature and limited follow-up duration. There was also a limited variety of contrast sensitivity testing conditions and modalities, including only photopic and not mesopic or scotopic lighting and a static image size in both Pelli-Robson and 
CamBlobs. The normative data base for both the PelliRobson and CamBolbs that were used are limited in the higher age bracket and more work will be required to adjust for reduction in contrast sensitivity associated with age alone. ${ }^{26}$ It may be helpful to incorporate contrast sensitivity testing as an end point in large scale studies to assess visual functional results such as determining in DR if earlier intervention would have any effect in preserving contrast sensitivity before manifest diabetic macular edema.

\section{Acknowledgments}

Dr Martin Ten Hove, Claudia Enderlein BHSc. Dr John Robson PhD, Precision Vision (www.Precision-Vision. com) provided the CamBlobs contrast sensitivity charts.

CamBlobs charts have now been superseded by a new version SpotChecksTM.

\section{Disclosure}

The authors report no conflicts of interest in this work.

\section{References}

1. Katz G, Levkovitch-Verbin H, Treister G, Belkin M, Ilany J, Polat U. Mesopic foveal contrast sensitivity is impaired in diabetic patients without retinopathy. Graefes Arch Clin Exp Ophthalmol. 2010;248 (12):1699-1703. doi:10.1007/s00417-010-1413-y

2. Sokol S, Moskowitz A, Skarf B, Evans R, Molitch M, Senior B. Contrast sensitivity in diabetics with and without background retinopathy. Arch Ophthalmol. 1985;103(1):51-54. doi:10.1001/ archopht.1985.01050010055018

3. Falsini B, Porciatti V, Scalia G, et al. Steady-state pattern electroretinogram in insulin dependent diabetics with no or minimal retinopathy. Doc Ophthalmol. 1989;73(2):193-200. doi:10.1007/BF00155037

4. Greenstein V, Sarter B, Hood D, Noble K, Carr R. Hue discrimination and cone pathway sensitivity in early diabetic retinopathy. Invest Ophthalmol Vis Sci. 1990;31:1008-1014.

5. Hegazy AI, Zedan RH, Macky TA, Esmat SM. Retinal ganglion cell complex changes using spectral domain optical coherence tomography in diabetic patients without retinopathy. Int J Ophthalmol. 2017;10 (3):427. doi:10.18240/ijo.2017.03.16

6. Varma R, Bressler NM, Suñer I, et al. Improved vision-related function after ranibizumab for macular edema after retinal vein occlusion: results from the BRAVO and CRUISE trials. Ophthalmology. 2012;119(10):2108-2118. doi:10.1016/j.ophtha.2012.05.017

7. Aiello LP, Beck RW, Bressler NM, et al. Rationale for the diabetic retinopathy clinical research network treatment protocol for centerinvolved diabetic macular edema. Ophthalmology. 2011;118(12):5-14. doi:10.1016/j.ophtha.2011.09.058

8. Baker CW, Glassman AR, Beaulieu WT, et al. Effect of initial management with aflibercept vs laser photocoagulation vs observation on vision loss among patients with diabetic macular edema involving the center of the macula and good visual acuity: a randomized clinical trial. JAMA. 2019;321(19):1880-1894. doi:10.1001/jama.2019.5790
9. Gruber N, Mosimann UP, Muri RM, Nef T. Vision and night driving abilities of the elderly. Traffic Inj Prev. 2013;14(5):477-485. doi:10.1080/15389588.2012.727510

10. Das A, McGuire PG, Rangasamy S. Diabetic macular edema: pathophysiology and novel therapeutic targets. Ophthalmology. 2015;122 (7):1375-1394. doi:10.1016/j.ophtha.2015.03.024

11. Hayreh SS. Ocular vascular occlusive disorders: natural history of visual outcome. Prog Retin Eye Res. 2014;41:1-25. doi:10.1016/j. preteyeres.2014.04.001

12. Ou WC, Brown DM, Payne JF, Wykoff CC. Relationship between visual acuity and retinal thickness during anti-vascular endothelial growth factor therapy for retinal diseases. Am J Ophthalmol. 2017;180:8-17. doi:10.1016/j.ajo.2017.05.014

13. Nieves-Moreno M, Martínez-de-la-casa JM, Cifuentes-Canorea P, et al. Normative database for separate inner retinal layers thickness using spectral domain optical coherence tomography in Caucasian population. PLoS One. 2017;12(7):e0180450. doi:10.1371/journal. pone. 0180450

14. Pelli DG, Robson JG, Wilkins AJ. The design of a new letter chart for measuring contrast sensitivity. Clin Vis Sci. 1988;2:187-99.37.

15. Elliott DB, Bullimore MA, Bailey IL. Improving the reliability of the Pelli-Robson contrast sensitivity test. Clin Vis Sci. 1991;6:471-475.

16. Griffin A, Cheng H, Robson J. Measuring contrast sensitivity using CambBlobs2 disposable paper charts in normal subjects. Invest Ophthalmol Vis Sci. 2017.

17. Robson J, Raman R, Jaisankar D, Sapkota RP, Pardhan S. Contrast sensitivity measured with illiterate single use printed paper charts to assess the severity of diabetic retinopathy. Invest Ophthalmol Vis Sci. 2017.

18. Canadian Medical Association. Section 12: Vision. Canadian Medical Association Driver's Guide. 8th ed. Toronto: Canadian Medical Association; 2012:48-52.

19. Fatehi N, Nowroozizadeh S, Henry S, Coleman AL, Caprioli J, Nouri-Mahdavi K. Association of structural and functional measures with contrast sensitivity in glaucoma. Am J Ophthalmol. 2017;178:129-139. doi:10.1016/j.ajo.2017.03.019

20. Ronnie G, Hemamalini A, Baskaran M, Ramesh SV, Raju P, Vijaya L. The Chennai glaucoma study: prevalence and risk factors for glaucoma in cataract operated eyes in urban Chennai Indian. $J$ Ophthalmol. 2010;58(3):243-245.

21. West SK, Rubin GS, Bowman AT, Munoz B, Bandeen-Roche K, Turano K. How does visual impairment affect performance on tasks of everyday life? The SEE project. Arch Ophthalmol. 2002;120 (6):774-780. doi:10.1001/archopht.120.6.774

22. Rubin GS, Bandeen-Roche K, Huang GH, et al. The association of multiple visual impairments with self-reported visual disability: SEE project. Invest Ophthalmol Vis Sci. 2001;42:64-72.

23. van Dijk HW, Verbraak FD, Kok PH, et al. Decreased retinal ganglion cell layer thickness in patients with type 1 diabetes. Invest Ophthalmol Vis Sci. 2010;51(7):3660-3665. doi:10.1167/iovs.095041

24. Stavrou EP, Wood JM. Letter contrast sensitivity changes in early diabetic retinopathy. Clin Exp Optom. 2003;86(3):152-156. doi:10.1111/j.1444-0938.2003.tb03097.x

25. Nixon DR, Flinn NA. Evaluation of contrast sensitivity and other visual function outcomes in diabetic macular edema patients following treatment switch to aflibercept from ranibizumab. Clin Ophthalmol. 2018;12:191-197. doi:10.2147/OPTH.S158268

26. Owsley C, Sekuler R, Siemsen D. Contrast sensitivity throughout adulthood. Vision Res. 1983;23(7):689-699. doi:10.1016/0042-6989 (83)90210-9 


\section{Publish your work in this journal}

Clinical Ophthalmology is an international, peer-reviewed journal covering all subspecialties within ophthalmology. Key topics include: Optometry; Visual science; Pharmacology and drug therapy in eye diseases; Basic Sciences; Primary and Secondary eye care; Patient Safety and Quality of Care Improvements. This journal is indexed on PubMed
Central and CAS, and is the official journal of The Society of Clinical Ophthalmology (SCO). The manuscript management system is completely online and includes a very quick and fair peer-review system, which is all easy to use. Visit http://www.dovepress.com testimonials.php to read real quotes from published authors. 